\title{
Introduction to High Energy Density Laboratory Astrophysics 2008
}

\author{
Tomek Plewa
}

Received: 21 May 2009 / Accepted: 21 May 2009 / Published online: 9 June 2009

(C) Springer Science+Business Media B.V. 2009

The 7th International Conference on High Energy Density Laboratory Astrophysics, HEDLA-08, was held in St. Louis, Missouri, on April 11-15, 2008. The meeting has been jointly organized by HEDLA and the American Physical Society, and shared its venue with the 1st International Conference on High Energy Density Physics. The conference was organized by Bruce Remington (LLNL, Chair), R. Paul Drake (Univ. of Michigan) Paul Bellan (Caltech), George Kyrala (LANL), Tomasz Plewa (Florida State Univ.), Sergey Lebedev (Imperial College), Hideaki Takabe (Osaka Univ.), Roberto Mancini (Univ. of Nevada-Reno), Ron Davidson (Princeton Univ.), Steve Rose (Imperial College), Kenichi Nomoto (Univ. of Tokyo), Jonathan Fortney (NASA-Ames), Edison Liang (Rice Univ.), Ryosuke Kodama (Osaka Univ.), Manuel Hegelich (LANL), Peter Norreys (RAL), Todd Ditmire (Univ. Texas-Austin), Barbara Jacak (SUNY-Stony Brook), and Tetsuo Hatsuda (Univ. of Tokyo).

The HEDLA-08 conference was a continuation of a series of biennial conferences first held in Pleasanton, California, in 1996, and reflected on progress made during recent years in designing, conducting, and analyzing the results of laboratory experiments, theoretical work, and computer simulations relevant to high-energy density (HED) astrophysics. The astrophysics setting for HED experiments is extremely diverse and includes jets, the mechanism of their generation and interaction with the ambient medium; stellar evolution with focus on turbulence, hydrodynamic instabilities, and mixing of nuclear species; radiative supersonic flows associated with stellar explosions and jets; radiative processes

T. Plewa $(\bowtie)$

Department of Scientific Computing, Florida State University,

Tallahassee, FL, USA

e-mail: tplewa@fsu.edu in plasma; equation of state and material properties; and last but not least magnetized and relativistic plasmas.

The HEDLA community continues enjoying support of and access to a wide range of the most technologically advanced experimental facilities including the $\mathrm{Z}$ Machine at the Sandia National Laboratory, the Omega laser at the University of Rochester, the LULI2000 laser at Ecole Polytechnique in France, and heavy ion beams accelerators. There is a strong expectation that new exciting results will soon become available thanks to OMEGA EP (Extended Performance) upgrade, and vigorous work has already started on designing experiments for the National Ignition Facility in the US and the Laser Megajoule in France. Also smaller but more accessible experimental facilities (Astra and Vulcan lasers at the Rutherford Appleton Laboratory, UK, Nike laser at Naval Research Laboratory, LFEX laser at the Institute of Laser Engineering, Osaka University, Japan, or ShenGuang family of lasers at the Research Center of Laser Fusion, China Academy of Engineering and Physics) are proving extremely useful in helping us understand physics of high energy density systems.

The conference has been organized into 13 oral sessions with about 60 review and original talks. There were two poster sessions providing ample time for just under 200 participants to discuss more than 100 presentations. This volume brings a smaller but representative subset of the HEDLA-08 meeting with 35 peer-reviewed papers.

I would like to thank the authors for their interest and contributions and the reviewers for investing their personal time and helping improving the original presentations. Special thanks go to the Editorial Office at Springer for their help and advise in managing my editorial work. Thank you!

Guest Editor

Tallahassee, May 2009 